\title{
Penetration of a coarse sand target by rigid projectiles
}

\author{
M. D. Hankins, B. C. Stoltz, K. L. Torres \& S. E. Jones \\ Department of Aerospace Engineering and Mechanics, \\ The University of Alabama, USA
}

\begin{abstract}
This paper presents a theory for the normal rigid body penetration of particulate media. This theory consists of two separate force regimes divided by a critical velocity at which the transition between the two regimes takes place. Also included in this theory is sliding friction, separated into two different regimes, one for the nose and one for the shank.

In order to verify this new penetration theory, a set of laboratory experiments was performed where 7075-T6 Aluminum projectiles were shot into coarse foundry sand. Utilizing the total penetration depth and impact velocity of each projectile in the test, along with known projectile geometry, analyses of the penetration events were completed. The results of these experiments and analyses, which confirm the required use of a friction coefficient on the shank, are reported.

Keywords: penetration mechanics, particulate media, projectile, rigid penetrator, critical velocity, friction coefficient, sliding friction.
\end{abstract}

\section{Introduction}

In two papers, Allen et al. [1,2] presented a theory for rigid body penetration into a sand target. The theory was supported by an extensive experimental matrix. The results indicated a radical change in the nature of the force law decelerating the projectile below some critical velocity. The critical velocity was attributed to the sound speed in the sand, but this is still an open question.

Later, Young [3] observed a similar trend in depth predictions for earthpenetrating projectiles in which a clear distinction was made for penetration 
depths with impact velocities above and below $200 \mathrm{ft} / \mathrm{sec}$. For impact velocities below $200 \mathrm{ft} / \mathrm{sec}$, penetration depth was linear in impact velocity.

These observations were applied to the development of another penetration equation. A series of laboratory scale experiments into "coarse foundry sand," a mixture of small particle silica, revealed the same projectile behavior. Two distinctly different force regimes decelerate the projectile, separated by a critical velocity. This resulted in two distinct branches to the penetration depth/impact velocity curve for a specific nose geometry. Working in these two branches of the curve are two different coefficients of friction, the effects of which are significant enough to require their inclusion in the theory. The results of a number of penetration tests with 0.164 " diameter 7075-T6 Aluminum with four different nose geometries are reported.

\section{Penetration of a confined particulate target}

The purpose of this analysis is to introduce a simple analytical model that will distribute the force on rod penetrators across the nose and the shank. As the projectiles move through the particulate medium, particles are moved laterally. These target particles also slide on the surface of the shank. Even with the low friction on the shank, there is a net axial force due to the interaction which is significant enough to be included in this analysis.

The force on the nose of the penetrator is due to a pressure that is developed by moving target material laterally as the penetrator makes its way through the target. This may include an increase in the average density, as the target material may become compacted in the process. This process of compaction has been used as a modelling tool [4], although the conventional approach to modelling this process is by cavity expansion methods (e.g., Luk and Forrestal [5]). All of these analyses lead to a velocity-squared pressure of the form

$$
P=\gamma \rho_{t} v_{n}^{2}+R
$$

where $\gamma$ is a dimensionless constant, $\rho_{t}$ is the target density, $\mathrm{R}$ is a constant with pressure dimensions, and $v_{n}$ is the magnitude of the component of the axial velocity vector in the direction of the normal to the surface of the nose (see Figure 1).

Let the nose of the projectile be described by $r=r(x)$, where $\mathrm{x}$ is the axis of the penetrator directed toward the aft-end. This coordinate system is attached to the projectile. Let sliding friction be present on the projectile nose and the shank. Take the sliding friction to be of the form

$$
f=\mu P
$$

where $\mu$ is the dimensionless coefficient of friction. This is a considerable over simplification of the friction that is present in a penetration event. Everything points to the fact that sliding friction decreases considerably with sliding speed [6]. In fact, this velocity dependence was used in an investigation of friction and wear on steel projectiles by concrete targets $[7,8]$. In an effort to minimize the complexity of this modelling effort, we have adopted eqn (2) with $\mu=$ const . 


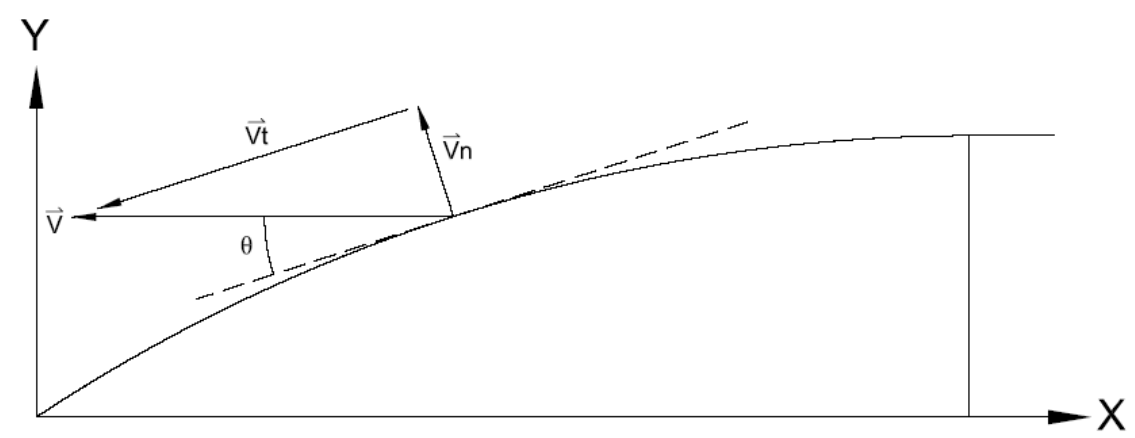

Figure 1: Velocity components.

The net axial resisting force on the nose of the penetrator can be found by taking the axial component due to $P$ and $f$ and integrating across the nose. Sparing some of the tedious details of this calculation, we can show that the total axial force of the nose of the penetrator is

$$
\left.F_{\text {nose }}=\pi a^{2} \mid \gamma \rho_{t}(N+\mu K) v^{2}+R(1+\mu M)\right]
$$

where $v=|\vec{v}|$ is the magnitude of the axial velocity vector and

$$
\begin{gathered}
N=\frac{2}{a^{2}} \int_{0}^{b} \frac{r r^{\prime 3}}{1+r^{\prime 2}} d x \\
K=\frac{2}{a^{2}} \int_{0}^{b} \frac{r r^{\prime 2}}{1+r^{\prime 2}} d x \\
M=\frac{2}{a^{2}} \int_{0}^{b} r d x
\end{gathered}
$$

are the nose shape factors for the nose of the penetrator. In these equations, $a$ is the radius of the shank of the penetrator (figure 2). The details that lead to eqn (3), along with eqns (4)-(6), have been sparingly presented. The reason for this is that they have appeared earlier (e.g., see Luk and Forrestal [5] and Jones et al. [9]).

The purpose of this paper is to add an additional force. As the particles of target material move laterally away from the nose to make way for the penetrator, a cavity is opened and the shank of the penetrator passes through it. Particles of sand sliding against the shank produce a friction force that helps to retard the motion of the penetrator. The sliding speed (tangential component) of the target particles against the shank is generally higher than it is across the nose. This would justify, at least on the average, the choice of two coefficients of friction, $\mu_{1}$ for the shank and $\mu_{0}$ for the nose. The remaining course of the modelling will use this approximation. The form of the friction in eqn (2) will still be the same, although we will use different coefficients of friction to account for the difference in tangential speed in the two regions. 


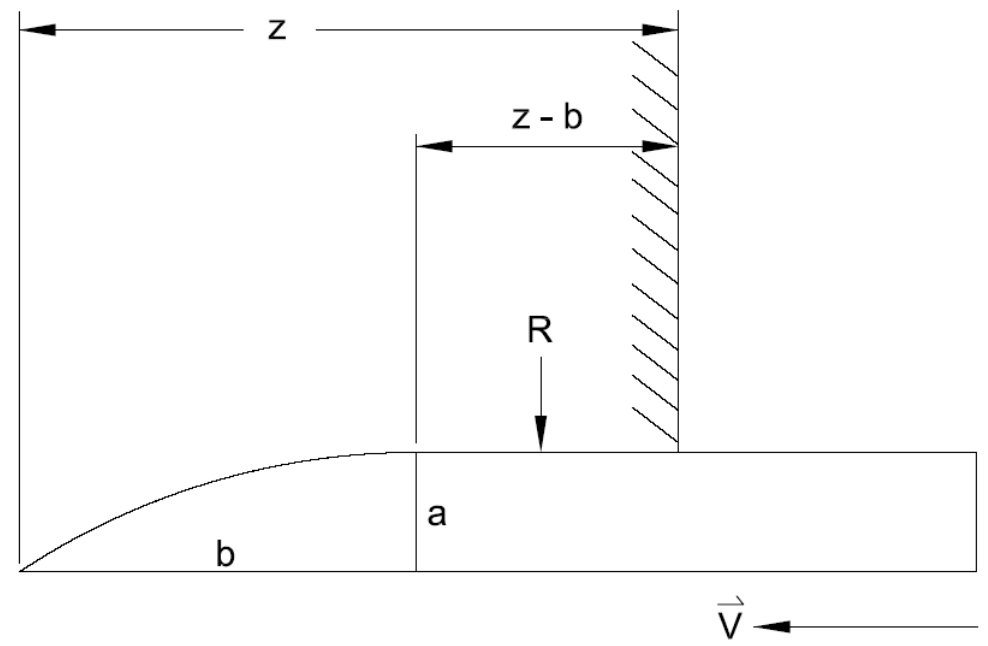

Figure 2: $\quad$ Partial immersion.

Using the reasoning of the previous paragraph, we turn to the resistance due to friction on the shank. There are two considerations: partial immersion of the shank in the target and complete immersion of the shank in the target. In the first case, partial immersion of the shank, we refer to Figure 2. As an approximation, we assume that the nose of the projectile can enter the target without appreciable loss of energy. For small projectiles, like those used in the next section of this paper, this is a good simplifying assumption. For larger projectiles, this will not be appropriate and the complication of the partial immersion of the nose will require additional consideration. For the present, the force retarding projectile motion due to partial friction on the shank is

$$
F_{\text {shank }}=\pi a^{2} \mu_{1} R(z-b) \text {. }
$$

Since the normal component of the axial velocity is equal to zero, the pressure on the shank is $P=R$, according to eqn (1). When the shank is fully immersed in the target, eqn (7) becomes

$$
F_{\text {shank }}=\pi a^{2} \mu_{1} R(L-b)
$$

where $\mathrm{L}$ is the overall length of the penetrator. These two forces, along with the force on the nose, eqn (3), with $\mu=\mu_{0}$, provide us with two separate cases for the penetration of sand.

For early time penetration, the resisting force has the form

$$
F=F_{\text {nose }}+F_{\text {shank }}=\pi a^{2}\left\lfloor\gamma \rho_{t}\left(N+\mu_{0} K\right) v^{2}+R\left(1+\mu_{0} M\right)\right\rfloor+\pi a^{2} \mu_{1} R(z-b)
$$

for $z<L$. The equation of motion for early penetration has the form

$$
m \dot{v}=-F
$$

where $\mathrm{F}$ is the net force given in eqn (9). Although nonlinear, this equation can be integrated by first changing the variables through $\dot{v}=v d v / d z$. Now, eqn (10) becomes the linear equation 


$$
\frac{d u}{d z}+\frac{2 \pi a^{2} \gamma \rho_{t}\left(N+\mu_{0} K\right)}{m} u=-\frac{2 \pi a^{2}\left(1+\mu_{0} M\right) R}{m}-\frac{4 \pi \mu_{1} R a}{m}(z-b)
$$

where $u=v^{2}$. An integrating factor for eqn (11) is $e^{k z}$ with

$$
k=\frac{2 \pi a^{2} \gamma \rho_{t}\left(N+\mu_{0} K\right)}{m}
$$

Multiplying eqn (11) by $e^{k z}$ and integrating leads to

$$
u=v^{2}=c_{1} e^{-k z}-\frac{2 \pi a^{2}\left(1+\mu_{0} M\right) R}{m k}+\frac{4 \pi \mu_{1} R a b}{m k}-\frac{4 \pi \mu_{1} R a}{m}\left(\frac{z}{k}-\frac{1}{k^{2}}\right)
$$

where $c_{1}$ is the constant of integration.

The initial condition used to evaluate the constant $c_{1}$ in eqn (13) is based on the approximation that there is negligible energy loss during penetration of the nose of the projectile. This simplification is $v=v_{0}$ when $z=b$, where the impact velocity is $v_{0}$. When this condition is applied, we get

$$
c_{1}=e^{k b}\left[v_{0}^{2}+\frac{2 \pi a^{2}\left(1+\mu_{0} M\right) R}{m k}-\frac{4 \pi \mu_{1} R a}{m k^{2}}\right] .
$$

When eqn (14) is used in eqn (13), we get

$$
\begin{aligned}
& e^{k(b-z)}\left[v_{0}^{2}+\frac{2 \pi a^{2}\left(1+\mu_{0} M\right) R}{m k}-\frac{4 \pi \mu_{1} R a}{m k^{2}}\right]-\frac{2 \pi a^{2}\left(1+\mu_{0} M\right) R}{m k} \\
& +\frac{4 \pi \mu_{1} R a b}{m k}-\frac{4 \pi \mu_{1} R a}{m}\left(\frac{z}{k}-\frac{1}{k^{2}}\right)=0
\end{aligned}
$$

which describes the motion of the projectile for partial immersion of the shank in the target. The final penetration depth, $z_{f}$, can be found for $z_{f}<L$ by solving for the roots of this transcendental equation. This equation persists in governing the motion until the shank is fully immersed in the target. If the impact velocity $v_{0}$ is high enough, then there will be a transition in the motion to a new equation of motion with $z=L$ in eqn (13). The new equation takes the form

$$
\frac{d u}{d z}+k u=\frac{-2 \pi a^{2} R\left(1+\mu_{0} M\right)-4 \pi \mu_{1} R a L_{0}}{m}
$$

where $L_{0}=L-b$, the shank length. This equation can be integrated by multiplying it by the integrating factor in eqn (12). This results in

$$
u=v^{2}=c_{2} e^{-k z}-\frac{2 \pi a^{2} R\left(1+\mu_{0} M\right)+4 \pi \mu_{1} R a L_{0}}{m k}
$$

where $c_{2}$ is the constant of integration.

To evaluate the constant of integration, we must match the branch of the penetration curve defined by eqn (17) with the branch defined by eqn (13). There is a transition velocity, say $v=\bar{v}$, at which the two branches match one another. The depth of penetration is $z=L$ at this transition point and 


$$
c_{2}=e^{k L}\left[\bar{v}^{2}+\frac{2 \pi a^{2} R\left(1+\mu_{0} M\right)+4 \pi \mu_{1} R a L_{0}}{m k}\right]
$$

At the same time, we know that

$$
\bar{v}^{2}=c_{1} e^{-k L}-\frac{2 \pi a^{2}\left(1+\mu_{0} M\right) R}{m k}+\frac{4 \pi \mu_{1} R a b}{m k}+\frac{4 \pi \mu_{1} R a}{m}\left(\frac{L}{k}-\frac{1}{k^{2}}\right)
$$

from eqn (13) with $c_{1}$ given in eqn (14). Now, we can combine eqns (18), (19), and (14) to get the penetration depth curve for impact velocities that exceed the transition velocity $\bar{v}$. After a number of algebraic simplifications, we can show that

$$
z=L+\frac{1}{k} \ln \left[\frac{e^{-k L_{0}\left(v_{0}^{2}+\frac{2 \pi a^{2}\left(1+\mu_{0} M\right) R}{m k}-\frac{4 \pi \mu_{1} R a}{m k^{2}}\right)+\frac{4 \pi \mu_{1} R a}{m k^{2}}}}{v^{2}+\frac{2 \pi a^{2}\left(1+\mu_{0} M\right) R}{m k}+\frac{4 \pi \mu_{1} R a L_{0}}{m k}}\right]
$$

For $v_{0}>\bar{v}$ in eqn (19). Together, eqns (13) and (20) comprise the theory for the penetration of sand presented in this paper. The reader will note that penetration depth according to eqn (13) will require numerical evaluation since this equation is transcendental. However, eqn (20) provides explicit evaluation of $z$ in terms of the current projectile speed $v$. Letting $v=0$, eqn (21) denotes the true equation for total penetration depth when $z_{f}>L$.

$$
z_{f}=L+\frac{1}{k} \ln \left[\frac{e^{-k L_{0}\left(v_{0}^{2}+\frac{2 \pi a^{2}\left(1+\mu_{0} M\right) R}{m k}-\frac{4 \pi \mu_{1} R a}{m k^{2}}\right)+\frac{4 \pi \mu_{1} R a}{m k^{2}}}}{\frac{2 \pi a^{2}\left(1+\mu_{0} M\right) R}{m k}+\frac{4 \pi \mu_{1} R a L_{0}}{m k}}\right]
$$

There are four free parameters in the model: $\mu_{0}, \mu_{1}, \gamma, R$. We anticipate that $\gamma$ may vary somewhat with nose geometry, while $\mu_{1}$ and $R$ should be properties of the target and penetrator materials. $\mu_{0}$ was assumed to be zero because the penetrator noses for this experiment had miniscule surface areas compared to that of the shank, and therefore little ability to contribute. A series of experiments were performed to validate the model and the results are presented in the next section.

\section{Results of penetration tests}

Utilizing the theory described in the previous section, experiments involving 7075-T6 Aluminum projectiles and coarse foundry sand were performed. The projectiles had an aspect ratio of 10:1, meaning that the total length, L, was ten times that of the shank diameter, $2 a$. Four different nose geometries were considered, the flat nose, or rod projectile, and three different ogive nose 
penetrators, the properties of which were evaluated in an earlier paper [ogive nose].

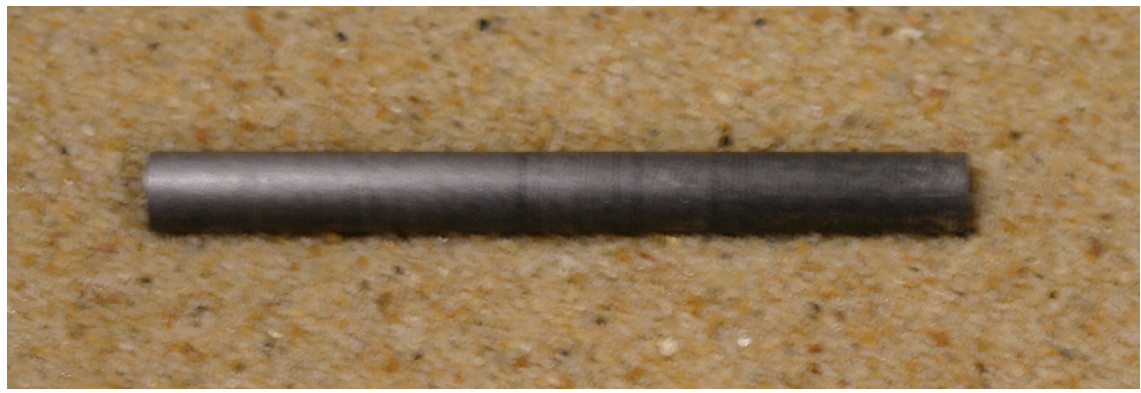

Figure 3: $\quad$ Particle size.

The reasons for choosing foundry sand as the target material were its ready availability and its contrast with the two other target media used in subsequent papers to aid in the validation of the new theory. The two other media were fine foundry sand [10] and alumina powder $\left(\mathrm{Al}_{2} \mathrm{O}_{3}\right)$ [11], both of which are denser and have a smaller particle size than the coarse sand. Figure 3 shows that despite the coarse sand's larger particle size, the projectile is not overmatched by the target particles. The difference between the two others appears to be that the alumina is a very high-friction medium, whereas the fine sand is an almost talclike, very low-friction environment. The coarse sand used here has a friction coefficient somewhere in between.

Before each test the target, a foot long, five inch diameter tube of sand, was compacted using a shake table to ensure a homogeneous environment that was identical every time a penetrator was shot. Flat nose projectiles were the first to be shot into the target material using a .167 caliber laboratory gun. A fast burning shotgun powder was used in rifle shell casings to accelerate the projectiles out of the gun tube and into the target. During the tests the impact velocity of each projectile was measured with parallel laser beams. Afterward, once the penetrator was recovered by digging cautiously into the target material until the back was located, the total penetration depth was measured. These experiments were repeated for ogive nose penetrators with "Caliber-radiusheads" of $0.5,3.0$, and 4.25. Recall that "Caliber-radius-head" refers to the ratio of ogive radius to diameter (e.g., see [5] or [9]).

These two pieces of data, impact velocity and penetration depth, for each test were all that was necessary to completely describe the penetration event. To reduce the effect of anomalous data points, more than ten tests per nose geometry were performed, each at a different velocity. Following this, the data was analyzed using the two coefficients of friction model. This analysis was performed for the flat nose penetrator with a computer program that, assuming $\mu_{0}=0$, tried various values of $\mu_{1}, \gamma$, and $R$ until the combination that showed the least deviation from experimental values was found. $R$ was then 
held constant while $\mu_{1}$ and $\gamma$ were found for the other nose geometries. The results of this analysis are contained in Table 1.

Table 1: $\quad$ Penetrator variables.

\begin{tabular}{|c|c|c|c|c|}
\hline & FLAT & OGIVE 0.5 & OGIVE 3 & OGIVE 4.25 \\
\hline$N$ & 1 & 0.5 & 0.1065 & 0.0761 \\
\hline$\gamma$ & 1.1 & 1.8 & 6.51 & 9.14 \\
\hline$R$ & $3 \mathrm{e} 6 \mathrm{~Pa}$ & $3 \mathrm{e} 6 \mathrm{~Pa}$ & $3 \mathrm{e} 6 \mathrm{~Pa}$ & $3 \mathrm{e} 6 \mathrm{~Pa}$ \\
\hline$\mu_{0}$ & 0.0 & 0.0 & 0.0 & 0.0 \\
\hline$\mu_{1}$ & 0.02 & 0.02 & 0.02 & 0.02 \\
\hline$v_{c}$ & $89.39 \mathrm{~m} / \mathrm{s}$ & $95.58 \mathrm{~m} / \mathrm{s}$ & $84.50 \mathrm{~m} / \mathrm{s}$ & $83.10 \mathrm{~m} / \mathrm{s}$ \\
\hline$L$ & $4.1656 \mathrm{~cm}$ & $4.1656 \mathrm{~cm}$ & $4.1656 \mathrm{~cm}$ & $4.1656 \mathrm{~cm}$ \\
\hline
\end{tabular}

N vs Gamma

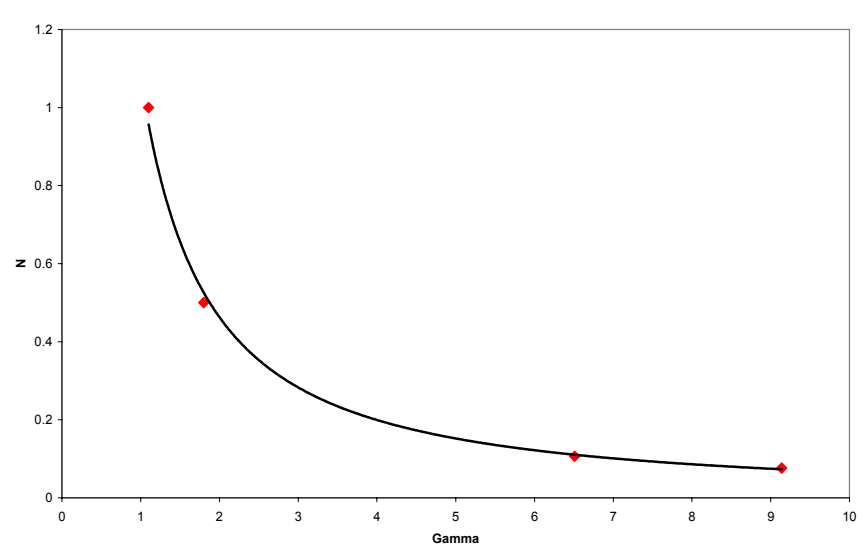

Figure 4: This graph shows the inverse relation of the nose factor, $\mathrm{N}$, to $\gamma$.

Some important things to note are that $\gamma$ was related inversely to nose factor $\mathrm{N}$ as demonstrated in Figure 4, and the coefficient of friction for the shank behaved as the model predicted. While $\mu_{0}=0, \mu_{1}$ varied only slightly. The last value on Table 1 , the critical or transitional velocity, is the velocity at which the back of the projectile is flush with the top of the target.

Figures 5-6 illustrate the value of these numbers. The first depicts a normalized penetration depth (total depth, $z_{f}$, divided by penetrator length) versus strictly the impact velocity, $v_{0}$, for the CRH 0.5 projectiles tested. The second shows the same normalized depth versus the available energy of the projectile at impact, $1 / 2 m v_{0}^{2}$. An inflection point is apparent on the graphs 
at $z / L=1$. This point, $v_{c}$, separates the two force regimes which decelerate the penetrator. In each figure, the theoretical normalized depth curve is presented twice. One curve is the predicted depth assuming a single coefficient of friction for the entire penetrator. The second, and as the reader will note, more accurate, curve utilizes the new model's two coefficients of friction. Similar graphs for each set of penetration tests exist.

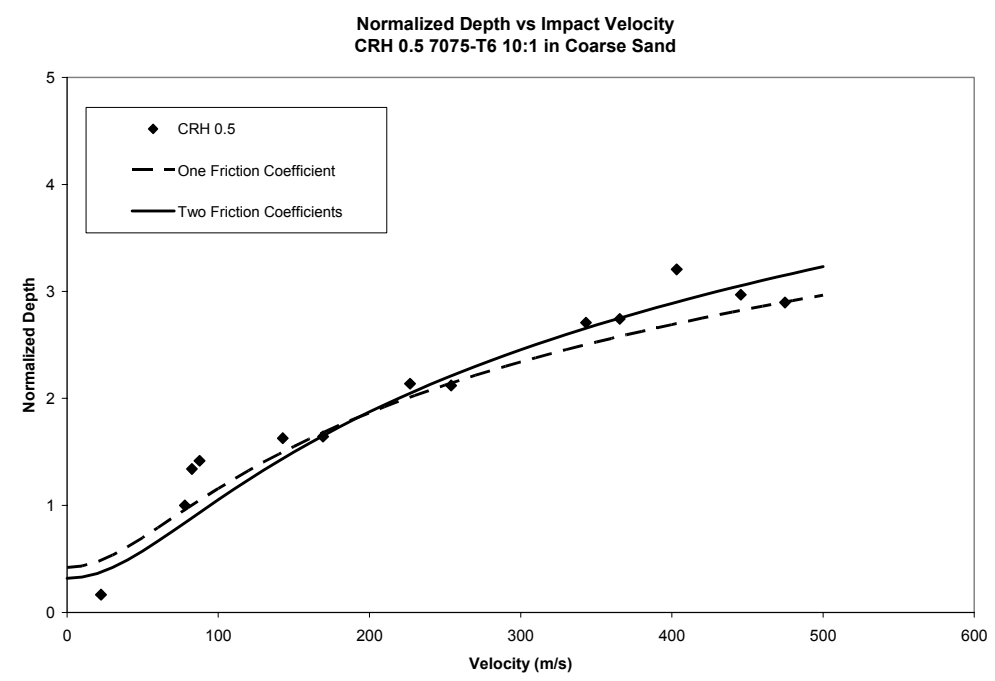

Figure 5: $\quad$ CRH 0.5 normalized depth vs. impact velocity.

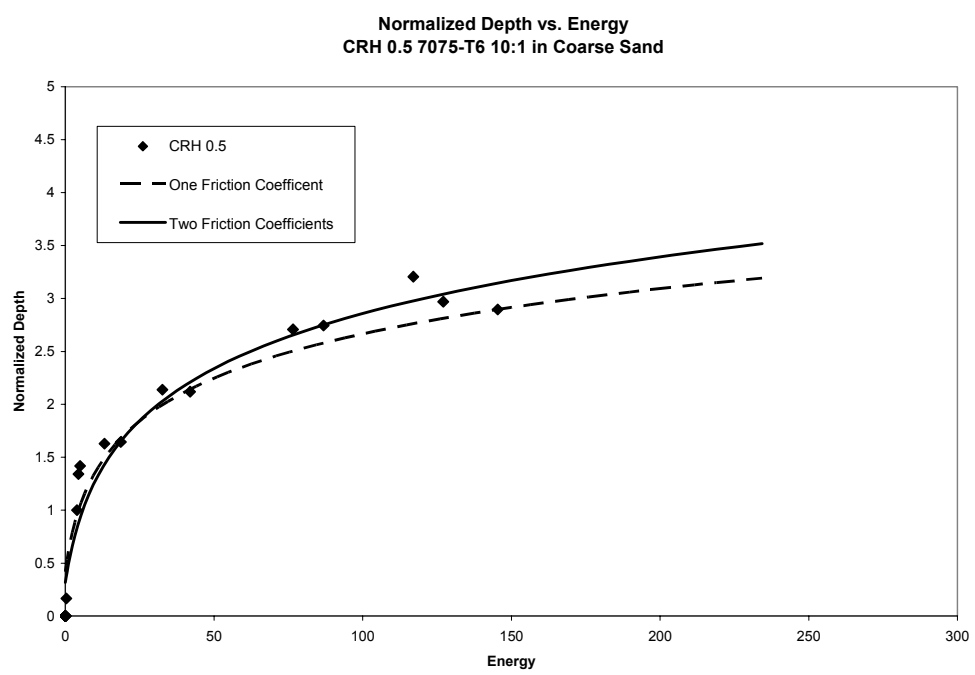

Figure 6: CRH 0.5 normalized depth vs. energy. 


\section{Conclusion}

The penetration model presented in this paper suggests the use of two different force regimes, separated by a critical velocity, to represent the penetration of a rigid projectile into a particulate medium. One force is dominant when the penetrator is only partially immersed, and the other takes over once full immersion has been achieved. Results of laboratory tests performed after the development of this theory nicely substantiate it with the behavior of the four free parameters of the model being very similar to that predicted by the theory.

\section{References}

[1] Allen, William A., Mayfield, Earle B., and Morrison, Harvey L., Dynamics of a Projectile Penetrating Sand. Journal of Applied Physics, 28(3), pp. 370-376, 1957.

[2] Allen, William A., Mayfield, Earle B., and Morrison, Harvey L., Dynamics of a Projectile Penetrating Sand, Part II. Journal of Applied Physics, 28(11), pp. 1331-1335, 1957.

[3] Young, C. Wayne, Depth of Prediction for Earth-Penetrating Projectiles. Proceedings of the ASCE, Journal of Soil and Foundations Division, 95, No. SM3, pp. 803-817, 1969.

[4] Nuckols, D. Zach and Jones, S. E., Strength Estimates for High Velocity Penetration of Geological Targets by the Compaction Ring Theory. ASME-PVP Proceedings, Problems Involving Thermal-Hydraulics, Liquid Sloshing and Extreme Loads on Structures, ed. F. J. Moody, San Deigo, pp. 173-182, 2004.

[5] Luk, V. K., and Forrestal, M. J., Penetration into Semi-Infinite, Reinforced Concrete Targets with Spherical and Ogive Nose Projectiles. International Journal of Impact Engineering, 6(4), pp. 291, 1987.

[6] Kragelskii, I. V., Friction and Wear, Butterworths, Inc., Washington, DC, 1965.

[7] Jones, S. E., Hughes, M. L., Toners, O. A., and Davis, R. N., A OneDimensional Analysis of Rigid-Body Penetration with High-Speed Friction. Proceedings of the Institute of Mechanical Engineers, Journal of Mechanical Engineering Science, 217(C), pp. 411-422, 2003.

[8] Davis Robert N., Neely, Amanda, M., and Jones, S. E., Mass Loss and Blunting During High-Speed Penetration. Proceedings of the Institute of Mechanical Engineers, Journal of Mechanical Engineering Science, 218(C), pp. 1053-1062, 2004.

[9] Jones, S. E., Rule, William K., Jerome, D. M., and Klug, R. T., On the Optimal Nose Geometry for a Rigid Penetrator. Computational Mechanics, 22(5), pp. 413-417, 1998.

[10] Stoltz, Brian C., Hankins, Melissa D., Torres, Karen L., and Jones, S. E., Penetration of a Fine-Grain Sand Target by a Rigid Projectile. Proceedings of the ASME-PVP Conference, 2006.

[11] Torres, Karen L., Jones, S. E., Penetration of a Particulate Target by a Rigid Projectile. Submitted for Publication, 2006. 